\title{
Effect of Static Stretching of the Biceps Brachii on Torque, Electromyography, and Mechanomyography During Concentric Isokinetic Muscle Actions
}

\author{
Tammy K. Evetovich, Natalie J. Nauman, Donovan S. Conley, And \\ JAY B. TODD
}

Human Performance Laboratory, Wayne State College, Wayne, Nebraska 68787.

\begin{abstract}
The purpose of this study was to determine the effect of an acute static stretching bout of the biceps brachii on torque, electromyography (EMG), and mechanomyography (MMG) during concentric isokinetic muscle actions. Eighteen (men, $n=10$; women, $n=8)$ adult subjects $(\mathrm{M} \pm S D$ age $=22.7$ \pm 2.8 years; weight $=78.0 \pm 17.0 \mathrm{~kg}$; height $=177.9 \pm 11.0$ $\mathrm{cm})$ performed maximal isokinetic $\left(30\right.$ and $\left.270^{\circ} \cdot \mathrm{s}^{-1}\right)$ forearm flexion strength testing on 2 occasions while EMG and MMG were recorded. Subjects were randomly assigned to stretching (STR) or nonstretching (NSTR) protocols before strength testing. Two-way ANOVAs with repeated measures revealed significantly $(p \leq 0.05)$ greater torque for NSTR $(\mathrm{M} \pm$ SEM $=36.9 \pm 3.3 \mathrm{~N} \cdot \mathrm{m})$ vs. STR $(35.2 \pm 3.3 \mathrm{~N} \cdot \mathrm{m})$, significantly greater MMG amplitude for STR vs. NSTR for $30^{\circ} \cdot \mathrm{s}^{-1}$ (STR $=93.5 \pm 14.4 \mathrm{mV} ;$ NSTR $=63.1 \pm 10.6 \mathrm{mV}$ ) and $270^{\circ} \cdot \mathrm{s}^{-1}$ $(\mathrm{STR}=207.6 \pm 35.6 \mathrm{mV} ; \mathrm{NSTR}=136.4 \pm 31.7 \mathrm{mV})$, and no difference in EMG amplitude. These results indicate that a greater ability to produce torque without prior stretching is related to the musculotendinous stiffness of the muscle rather than the number of motor units activated. This suggests that performing activities that reduce muscle stiffness (such as stretching), may be detrimental to performance.
\end{abstract}

Key Words: autogenic inhibition, musculotendinous stiffness, acute

Reference Data: Evetovich, T.K., N.J. Nauman, D.S. Conley, and J.B. Todd. Effect of static stretching of the biceps brachii on torque, electromyography, and mechanomyography during concentric isokinetic muscle actions. J. Strength Cond. Res. 17(3):484-488. 2003.

\section{Introduction}

Stretching is commonly included as part of the Swarm-up routine with the principal intent to prevent injuries and improve muscular performance. Recent research has indicated that acute static stretching of a muscle prior to maximal muscular performance may negatively impact strength production for isometric (7), isotonic (11), and isokinetic (12) muscle actions. Hypotheses attempting to explain the deleterious affects of acute stretching include reduced stiffness of the musculotendinous unit, resulting in a less effective transfer of force from the muscle to the lever (11, 16) and/or greater autogenic inhibition, and therefore, fewer motor units activated in a stretched muscle $(2$, 10, 14, 15).

Mechanomyography (MMG) and electromyography (EMG) may facilitate a more thorough examination of these hypotheses. MMG is the recording of the muscular vibrations produced by active muscle $(3,4$, 8,13 ) and Barry and Cole (3) have suggested that the MMG signal can be used "... as a monitor of muscle stiffness." A study by Orizio et al. (13) supports this contention in which they reported that, during isometric muscle actions of the biceps brachii, a high level of torque production, and therefore a stiffer muscle, resulted in decreased MMG amplitude values when compared with lower levels of torque production. It was suggested (13) that the reduction in MMG amplitude may have been due to reduced muscle compliance. Nevertheless, previous studies have not reported the use of MMG to study stretching-related decrements in muscular performance and its relationship to musculotendinous stiffness. In addition, few studies (7) have shown that the number of motor units and the level of muscle activation, as measured by EMG, is reduced during maximal muscle actions of a stretched muscle. Given that Gordon and Holbourn (9) have stated that MMG reflects the "mechanical counterpart" of motor unit electrical activity as measured by EMG, simultaneous measurements of these signals may provide information about the effect of stretching on the mechanical and electrical events leading to 
muscular contraction. Therefore, the purpose of this study was to determine the effect of acute static stretching of the biceps brachii on torque, EMG, and MMG during concentric isokinetic muscle actions. Based on past research, we hypothesize in the present study that there will be reduced torque, decreased EMG amplitude (due to greater autogenic inhibition), and greater MMG amplitude (indicating less muscle stiffness) in the stretched vs. nonstretched biceps brachii muscle.

\section{Methods}

\section{Experimental Approach to the Problem}

Although investigators have proposed that stretching has a detrimental effect on maximal torque production by i) reducing the number of motor units activated and ii) decreasing muscular stiffness, no previous studies have conclusively determined that these 2 factors are affected during muscular performance. In this study, we wanted to measure the effect of stretching on the degree of muscle activation (using EMG) and muscle stiffness (using MMG) in an upper-body muscle during maximal dynamic torque production. Thus, we measured the strength, EMG, and MMG in a stretched vs. nonstretched muscle during concentric isokinetic muscle actions of the biceps brachii.

\section{Subjects}

Eighteen college-aged $(\mathrm{M} \pm S D$ age $=22.7 \pm 2.8$ years; weight $=78.0 \pm 17.0 \mathrm{~kg}$; height $=177.9 \pm 11.0 \mathrm{~cm}$ ) men $(n=10)$ and women $(n=8)$ participated in the study. The study was approved by the Institutional Review Board of the college, and each participant completed a health-history questionnaire form and signed an informed consent prior to testing. The subjects were volunteers from exercise science courses at the college and all subjects indicated on the health history questionnaire meeting the minimum requirements for a moderate level of aerobic activity as classified by the American College of Sports Medicine (1) on a regular basis (at least 3 times a week). In addition, 9 of the subjects reported that they had been participating in a recreational weight-training program with no indication of a history of competitive weightlifting.

\section{Strength Testing Protocol}

Each subject performed maximal isokinetic forearm flexion strength testing with the nondominant arm on 2 occasions separated by at least 48 hours while EMG and MMG were recorded. The isokinetic torque was measured on a calibrated Cybex II dynamometer at 2 different velocities $\left(30\right.$ and $270^{\circ} \cdot \mathrm{s}^{-1}$ ) with the subjects in a supine position on the Cybex II upper-body exercise table. Prior to testing, subjects were randomly assigned to either a stretching (STR) or nonstretching (NSTR) protocol. Three maximal muscle actions were then recorded at each velocity and the highest torque at each velocity was used for analysis. Subjects were instructed to perform maximally during the forearm flexion phase and relax during the forearm extension phase. Two minutes of rest were allowed between testing at each velocity.

\section{STR and NSTR Protocols}

The STR protocol was based on the stretching regimen conducted in the investigation of Nelson et al. (12) and consisted of 3 different static stretches designed to target the forearm flexor muscle group of the nondominant arm. Stretches consisted of i) placing the palm of the hand on a table with the glenohumeral joint externally rotated and the fingers directed posterior, ii) the arm horizontally abducted with the palm flat against a wall while applying resistance with the subject standing erect, and iii) a researcher actively stretching the subject's nondominant arm to a horizontally abducted position with the thumbs pointed toward the floor and the shoulder internally rotated at a point in which the subject indicated they had reached their full range of motion. Each stretch was held for 30 seconds and was repeated 4 times with 15 seconds between stretches. For the NSTR protocol, subjects did not stretch or perform physical activity prior to strength testing.

\section{EMG}

A bipolar (7.62 cm center-to-center) surface electrode (Quinton Quick Prep Ag- $\mathrm{AgCl}$ chloride, recording diameter $=1.5 \mathrm{~cm}$; Quinton, Bothello, WA) arrangement was placed over the nondominant biceps brachii muscle. The interelectrode distance was selected to accommodate the MMG sensor. The recording electrodes were placed over the belly of the muscle approximately midway between the axillary fold and the midpoint of the cubital fossa. The reference electrode was placed over the volar arch. Interelectrode impedance was kept below 5,000 ohms by abrasion of the skin. The EMG signal was amplified (gain: $\times 1,000$ ) using a differential amplifier (EMG 100, Biopac System, Inc., Santa Barbara, CA). To assure consistent placement of the electrodes throughout the study, a permanent marker was used on the skin to outline the electrodes.

\section{$M M G$}

The MMG signal was detected by a piezoelectric crystal contact sensor (Hewlett-Packard, Andover, MA; model \#21050A; bandwidth $0.02-2,000 \mathrm{~Hz}$ ) that was placed over the biceps brachii between the two EMG electrodes. A stabilizing ring, double-sided adhesive tape, and paper tape were used to ensure consistent contact pressure of the sensor.

\section{Signal Processing}

The amplitude values (rms) were calculated for a time period (2.00 seconds and 0.22 second for 30 and $270^{\circ} \cdot \mathrm{s}^{-1}$, respectively) that corresponded to a $60^{\circ}$ range 
Table 1. The mean ( $\pm S E M)$ torque, EMG amplitude, and MMG amplitude values for the stretching (STR) vs. nonstretching (NSTR) protocols.

\begin{tabular}{ccc}
\hline & STR & NSTR \\
\hline $\begin{array}{c}\text { Torque }(\mathrm{N} \cdot \mathrm{m}) \\
30^{\circ} \cdot \mathrm{s}^{-1}\end{array}$ & $49.5 \pm 4.1$ & $50.4 \pm 4.1$ \\
$270^{\circ} \cdot \mathrm{s}^{-1}$ & $20.9 \pm 2.5$ & $23.4 \pm 2.5$ \\
$\mathrm{EMG}(\mu \mathrm{V})$ & & \\
$30^{\circ} \cdot \mathrm{s}^{-1}$ & $925.1 \pm 81.0$ & $1,018.2 \pm 107.6$ \\
$270^{\circ} \cdot \mathrm{s}^{-1}$ & $784.9 \pm 90.1$ & $895.0 \pm 77.3$ \\
$\mathrm{MMG}(\mathrm{mV})$ & & \\
$30^{\circ} \cdot \mathrm{s}^{-1}$ & $93.5 \pm 14.4$ & $63.1 \pm 10.6$ \\
$270^{\circ} \cdot \mathrm{s}^{-1}$ & $207.6 \pm 35.6$ & $136.4 \pm 31.7$ \\
\hline
\end{tabular}

of motion from approximately $60-120^{\circ}$ of flexion at the elbow. The muscle action with the highest peak torque was used as the representative score for both EMG and MMG at each velocity. When selecting the EMG signal, the corresponding MMG signal was automatically selected so that the analyzed EMG and MMG signals were of the same time frame. The EMG and MMG signals were filtered at $10-500$ and $5-100 \mathrm{~Hz}$, respectively, and sampled at 1,000 points per second. Previous test-retest reliability from our laboratory for torque, EMG amplitude, and MMG amplitude during maximal CON isokinetic muscle actions indicated that, for men and women subjects measured 48 hours apart, the intraclass correlation coefficients $(R)$ were in the ranges of $0.87-0.93,0.97-0.99$, and $0.80-0.92$, respectively, with no significant differences between mean values for test vs. retest at velocities ranging from 30 to $300^{\circ} \cdot \mathrm{s}^{-1}$.

\section{Statistical Analyses}

Separate 2 (velocity $=30$ and $\left.270^{\circ} \cdot \mathrm{s}^{-1}\right) \times 2$ (protocol $=$ STR and NSTR) ANOVAs with repeated measures were used to determine differences in torque, EMG amplitude, and MMG amplitude. An alpha of $p \leq 0.05$ was considered statistically significant for all analyses.

\section{Results}

Table 1 displays the mean torque (N.m), EMG amplitude $(\mu \mathrm{V})$, and MMG amplitude $(\mathrm{mV})$ data $( \pm$ SEM) for STR vs. NSTR at 30 and $270^{\circ} \cdot \mathrm{s}^{-1}$.

\section{Torque}

There was no significant 2-way interaction but a significant main effect for protocol and velocity. On examination of the mean values, the results revealed significantly $(p<0.05)$ greater torque for NSTR ( $\mathrm{M} \pm$ SEM $=36.9 \pm 3.3 \mathrm{Nm}$ ) compared with STR (35.2 \pm $3.3 \mathrm{~N} \cdot \mathrm{m})$ collapsed across velocity and for $30^{\circ} \cdot \mathrm{s}^{-1}(50.0$ $\pm 4.1 \mathrm{~N} \cdot \mathrm{m})$ compared with $270^{\circ} \cdot \mathrm{s}^{-1}(22.2 \pm 2.5 \mathrm{~N} \cdot \mathrm{m})$ collapsed across protocol.

\section{EMG}

The 2-way ANOVA revealed no significant interaction or main effects, indicating that there was no change in EMG amplitude as a result of the stretching protocol.

\section{MMG}

The results revealed a significant protocol by velocity (2-way) interaction. Subsequent analyses included paired $t$-tests for each velocity for STR vs. NSTR. The results indicated significantly greater MMG amplitude for STR vs. NSTR for $30^{\circ} \cdot \mathrm{s}^{-1}$ (STR $=93.5 \pm 14.4 \mathrm{mV}$; NSTR $=63.1 \pm 10.6 \mathrm{mV})$ and $270^{\circ} \cdot \mathrm{s}^{-1}(\mathrm{STR}=207.6$ $\pm 35.6 \mathrm{mV}$; NSTR $=136.4 \pm 31.7 \mathrm{mV}$ ). These results represented 34.3 and $32.5 \%$ declines in MMG amplitude from STR to NSTR for 270 and $30^{\circ} \cdot \mathrm{s}^{-1}$, respectively.

\section{Discussion}

Nelson et al. (12) have indicated that "the deleterious impact of stretching activities on maximal torque production might be limited to movements performed at relatively slow velocities" during concentric isokinetic muscle actions of the knee extensors. The impact of stretching on maximal torque production was not velocity specific in the present study in that stretching hindered the full capabilities of the biceps brachii at both a slow $\left(30^{\circ} \cdot \mathrm{s}^{-1}\right)$ and fast $\left(270^{\circ} \cdot \mathrm{s}^{-1}\right)$ velocity. The reason for the contradictory findings between those of Nelson et al. (12) and the present study is not known, but may be a function of the muscle group (upper body vs. lower body) studied.

The results of the present study support our hypothesis and are in agreement with previous investigations $(7,11,12)$ that reported stretching negatively impacts the torque-producing capabilities of a muscle. The reasons given for this phenomenon have been largely attributed to two factors: i) less musculotendinous stiffness and ii) a reduced ability to recruit motor units. With regard to musculotendinous stiffness, e.g., Wilson et al. (16) used an oscillation technique during isometric bench press muscle actions and demonstrated that less musculotendinous stiffness, like that observed in a stretched muscle, may interfere with the ability to transfer force from the muscle to the skeletal system. In addition, Wilson et al. (16) suggested that a less stiff muscle should conceivably create a less than optimal length and velocity condition. More specifically, these investigators (16) stated that a stiffer musculotendinous unit should result in "a greater length of the contractile component and a reduced contractile component shortening velocity," which ultimately enhances the force-velocity and length-tension relationship, resulting in greater torque production. Furthermore, investigators have hypothesized that a reduction in torque due to stretching may be a function of greater autogenic inhibition and a de- 
creased ability to recruit motor units. This hypothesis is based on investigations $(2,10,14,15)$ that have reported a decrease in muscle activation and excitability during stretching as measured by the Hoffman reflex. Although these hypotheses have been put forth to explain the detrimental affects of stretching on muscular performance, to our knowledge, only one study (7) has attempted to quantify the degree of muscle activation and the level of musculotendinous stiffness in a stretched vs. nonstretched muscle during maximal torque production. In the present study, we employed 2 techniques, EMG and MMG, to examine these factors.

Although it has been hypothesized that stretching a muscle results in a reduced activation level, it is not clear as to how long the depression in motor neuron excitability may persist after stretching, what type of stretching protocol may elicit the reduction, and/or whether stretching-induced autogenic inhibition would carry over during actual muscular performance. If in fact stretching does have sustainable affects on muscle activation, we hypothesized in the present study that there would be greater EMG amplitude for NSTR vs. STR. The results of the present study did not support our hypothesis in that there was no reduction in muscle activation with stretching as evidenced by the nonsignificant difference in EMG amplitude for STR vs. NSTR. These results are not in accordance with Fowles et al. (7), who measured the EMG during maximal isometric muscle actions of the plantar flexors 5, 15, 30, 45, and 60 minutes after prolonged stretching (30 minutes total) and found that muscle activation was depressed immediately poststretching but recovered within 15 minutes. In the present study, the isokinetic muscle actions were performed approximately 5 minutes after stretching (to allow time for placement of the electrodes and sensor) and yet the EMG amplitude was not different between STR and NSTR. It is difficult to compare the present study with that of Fowles et al. (7), however, given that the length and intensity of the stretching protocol was not similar. In fact, these investigators (7) acknowledged that the duration of stretch performed in their study was more similar to the stretching protocols used in animal experiments and, therefore, "may have limited application to sport stretching performed in conjunction with athletic performance."

Previous studies have indicated that a stiffer muscle may possibly attenuate muscular vibrations and result in lower MMG amplitude values while a more compliant muscle would result in reduced stiffness, increased muscle fiber oscillations, and greater MMG amplitude $(3,5,6,13)$. Thus, we hypothesized in the present study that there would be greater MMG amplitude for STR (reduced stiffness) when compared with NSTR (greater stiffness) during maximal torque production. The results of the present study support our hypothesis in that there was greater MMG amplitude for STR vs. NSTR at both fast and slow velocities. These findings are in agreement with previous investigations $(11,12)$ in which the researchers concluded that a greater ability to produce torque without prior stretching is related to increased musculotendinous stiffness of the muscle.

\section{Practical Applications}

The results of the present investigation demonstrate that stretching immediately prior to muscular performance negatively impacts the torque-producing capabilities of a muscle at both slow and fast velocities for an upper-body movement. This suggests that, for many types of athletic performances that involve maximal muscle actions of the upper body, stretching may not be warranted and in fact may be detrimental. In addition, the greater strength capabilities observed without stretching is likely due in large part to increased muscular stiffness. In our study, stretching was the means by which muscular stiffness was influenced; however, athletes frequently participate in other activities prior to performance such as warming up with calisthenics, cycling, or light resistance training. It is possible that these other activities could influence the degree of muscular stiffness and ultimately compromise the full capabilities of the muscle. Future studies should examine whether the changes that may be induced by warm-up exercises (i.e., increased muscle temperature and blood flow) affects muscle stiffness.

\section{References}

1. American College of Sports Medicine. ACSM's Guidelines for Exercise Testing and Prescription. Baltimore: Lippincott Williams and Wilkins, 2000.

2. Avela, J., H. KYrolainen, And P. Komi. Altered reflex sensitivity after repeated and prolonged passive muscle stretching. J. Appl. Physiol. 86:1283-1291. 1999.

3. BARRY, D.T., AND N.M. COLE. Fluid mechanics of muscle vibrations. Biophys. J. 53:899-905. 1988.

4. BARRY, D.T., AND N.M. COLE. Muscle sounds are emitted at the resonant frequencies of skeletal muscle. IEEE Biomed. Eng. 37: 525-531. 1990.

5. Evetovich, T., T. Housh, G. Johnson, J. Stout, D. Smith, And K. EBERSOLE. Mechanomyographic responses to concentric isokinetic muscle contractions. Eur. J. Appl. Physiol. 75:166-169. 1997.

6. Evetovich, T., T. Housh, G. Johnson, J. Stout, D. Smith, K. Ebersole, And S. Perry. Gender comparisons of the mechanomyographic responses to maximal concentric and eccentric isokinetic muscle actions. Med. Sci. Sports Exerc. 30:1697-1702. 1998.

7. FOWles, J.R., D.G. SAlE, AND J.D. MACDOUGALl. Reduced strength after passive stretch of the human plantar flexors. J. Appl. Physiol. 89:1179-1188. 2000.

8. FrangIOnI, J.V., T.S. KWAn-GetT, L.E. DobrunZ, And T.A. McMAHON. The mechanism of low frequency sound production in muscle. Biophys. J. 51:775-783. 1987. 
9. Gordon, G., AND H. HolbourN. The sounds from single motor units in a contracting muscle. J. Physiol. 107:456-464. 1948.

10. Guissard, N., J. Duchateau, and K. Hainaut. Muscle stretching and motoneuron excitability. Eur. J. Appl. Physiol. 58:47-52. 1988.

11. KokKonen, J., A.G. Nelson, And A. Cornwell. Acute muscle stretching inhibits maximal strength performance. Res. Q. Exerc. Sport 69:411. 1998.

12. Nelson, A., I. Guillory, A. Cornwell, and J. KoKKOnen. Inhibition of maximal voluntary isokinetic torque production following stretching is velocity-specific. J. Strength Cond. Res. 15: 241-246. 2001.

13. Orizio, C., R. Perini, ANd A. Veicsteinas. Changes of muscular sound during sustained isometric contraction up to exhaustion. J. Appl. Physiol. 66:1593-1598. 1989.
14. Thigpen, L.K., T. Moritani, R. Thiebaud, And J.L. Hargis. The acute effects of static stretching on alpha motoneuron excitability. In: Biomechanics. IX-A. D.A. Winter, R.W. Norman, R.P. Wells, K.C. Hayes, and A.E. Patla, eds. Champaign, IL: Human Kinetics, 1985. pp. 352-357.

15. VujnOVICH, A.L., AND N.J. DAwsON. The effect of therapeutic muscle stretch on neural processing. J. Orthop. Sports Phys. Ther. 20:145-153. 1994.

16. Wilson, G.J., A.J. MurPHY, AND J.F. PryOR. Musculotendinous stiffness: Its relationship to eccentric, isometric, and concentric performance. J. Appl. Physiol. 76:2714-2719. 1994.

Address correspondence to Tammy K. Evetovich, taeveto1@wsc.edu. 